PROCEEDINGS OF THE

AMERICAN MATHEMATICAL SOCIETY

Volume 131, Number 1, Pages 17-20

S 0002-9939(02)06490-0

Article electronically published on June 12, 2002

\title{
SYMMETRIC PRESENTATIONS OF ABELIAN GROUPS
}

\author{
MIKLÓS ABÉRT \\ (Communicated by Stephen D. Smith)
}

\begin{abstract}
We characterise the abelianisation of a group that has a presentation for which the set of relations is invariant under the full symmetric group acting on the set of generators. This improves a result of Emerson.
\end{abstract}

Symmetric presentations have been investigated for a long time (see Cox , Eme, Bee and $[\mathrm{CM})$. Recently the focus of interest has been in constructing the finite simple groups in this way. The sporadic case is investigated in $\mathrm{Cu} 1$, Cu2, CHB and $[\mathrm{BC}]$ while the Lie-type case is treated in [CR], CRW], $\mathrm{RC}$ ] and [CHLR].

Definition 1. Let $G=(F \mid R)$ be a presentation of the group $G$. We call this presentation symmetric if $R$ is invariant under the action of the full symmetric group permuting the generating set $F$.

Definition 2. Let $G=\left\langle g_{1}, g_{2}, \ldots, g_{n}\right\rangle$ be an arbitrary group. We say that the $g_{i}$ 's symmetrically generate $G$ if the presentation belonging to that generating set is symmetric.

In this paper we determine which finite Abelian groups can be symmetrically generated. This can be considered as a 'test of symmetric generation' for an arbitrary group $G$, since if $G$ is symmetrically generated, then its abelianisation $G / G^{\prime}$ is symmetrically generated as well. Our result is a generalisation of the results of Emerson (see [Eme], who gives the canonical form of the abelianisation of a group that can be defined by a single relation and all of its permutations. He expresses the canonical form from the exponent sums of the single relation. We make use of the methods of Emerson and obtain a somewhat surprising result.

Theorem 1. Let $G$ be a finite Abelian group. Then $G$ can be symmetrically generated by $n>2$ elements if, and only if, there are positive integers $a, b, c$ such that

(i) $G=Z_{a} \times Z_{a b}^{n-2} \times Z_{a b c}$, and

(ii) $\operatorname{gcd}(b, c) \mid n$.

Comment. The case $n=2$ is trivial, since $Z_{a b} \times Z_{a} \simeq\langle x, y| x^{a b}=y^{a b}=\left(x y^{-1}\right)^{a}=$ $\left.x^{-1} y^{-1} x y=1\right\rangle$.

Received by the editors March 26, 2001 and, in revised form, August 2, 2001.

2000 Mathematics Subject Classification. Primary 20F05, 20 K01.

Key words and phrases. Presentations, Abelian groups.

This research was supported by the Hungarian National Grant T29132.

(C)2002 American Mathematical Society 
Proof. First we prove that if $G$ is symmetrically generated, then (i) and (ii) hold.

Let $G=\left\langle g_{1}, g_{2}, \ldots, g_{n}\right\rangle$ be a symmetric generating set of $G$. For an arbitrary integer $F$ that will be specified later, let us define $h_{1}, \ldots, h_{n} \in G$ in the following way:

$$
\begin{aligned}
& h_{1}=g_{1}, \\
& h_{i}=i g_{1}-\sum_{j=1}^{i} g_{j} \quad(1<i<n), \\
& h_{n}=F g_{1}-\sum_{j=1}^{n} g_{j} .
\end{aligned}
$$

We claim that there is an $F$ for which $h_{1}, h_{2}, \ldots, h_{n}$ is a base of $G$. We have to prove that $G=\left\langle h_{1}, h_{2}, \ldots, h_{n}\right\rangle$ and that if $\sum_{j=1}^{n} r_{j} h_{j}=0$, then $r_{j} h_{j}=0$ for all $j$.

Now $G=\left\langle h_{1}, h_{2}, \ldots, h_{n}\right\rangle$ directly follows from the fact that the transformation matrix of $g_{i} \rightarrow h_{i}$,

$$
M=\left(\begin{array}{cccccc}
1 & 0 & 0 & \ldots & 0 & 0 \\
1 & -1 & 0 & \ldots & 0 & 0 \\
2 & -1 & -1 & \ldots & 0 & 0 \\
\vdots & \vdots & \vdots & \ddots & \vdots & \vdots \\
n-2 & -1 & -1 & \ldots & -1 & 0 \\
F-1 & -1 & -1 & \ldots & -1 & -1
\end{array}\right)
$$

has $\operatorname{det}(M)=(-1)^{n-1}$, so it can be inverted over $\mathbb{Z}$.

Let us assume that $\sum_{j=1}^{n} r_{j} h_{j}=0$ and there is an index $k<n$, for which $r_{k} h_{k} \neq 0$. Let $k$ be the largest such index.

First suppose that $1<k<n$. Taking the nonzero parts of the sum $\sum_{j=1}^{n} r_{j} h_{j}=$ 0 and using the definition of $h_{i}$ we get $\sum_{j=1}^{n} s_{j} g_{j}=0$ where $s_{k}=-\left(r_{k}+r_{n}\right)$, $s_{k+1}=-r_{n}$. Now using the symmetry of the generating set $g_{1}, g_{2}, \ldots, g_{n}$ of $G$, and applying the transposition $(k, k+1)$, finally taking the difference of the two zero sums we get $r_{k}\left(g_{k}-g_{k+1}\right)=0$. Using the symmetry again we get $r_{k}\left(g_{i}-g_{j}\right)=0$ for all $i \neq j$. But then $0 \neq r_{k} h_{k}=r_{k} \sum_{j=1}^{k}\left(g_{1}-g_{j}\right)=0$, which is a contradiction.

So we have to find an $F$ for which $h_{1}$ and $h_{n}$ are independent and we will be done.

Let $p$ be the order of $h_{1}=g_{1}$. Then for all $g \in G, p g=0$ because of the symmetry. So $\left\langle h_{1}\right\rangle$ is a cyclic subgroup of maximal order in $\left\langle h_{1}, \sum_{j=1}^{n} g_{j}\right\rangle$ and, as such, it has a direct complement. So we have an integer $F$ such that

$$
\left\langle h_{1}, \sum_{j=1}^{n} g_{j}\right\rangle=\left\langle h_{1}\right\rangle \oplus\left\langle F h_{1}-\sum_{j=1}^{n} g_{j}\right\rangle .
$$

We have proved that $h_{1}, h_{2}, \ldots, h_{n}$ is a base of $G$.

Now we show (i) and (ii). Let $q$ be the order of $h_{2}$. Then $q\left(g_{1}-g_{2}\right)=0$. Using the symmetry we get $q\left(g_{i}-g_{j}\right)=0$, from which $q h_{k}=q \sum_{j=1}^{k}\left(g_{1}-g_{j}\right)=0$ follows for all $1<k<n$. On the other hand, let us assume that there is a $z \in \mathbb{Z}$, for which $z h_{k}=0$ for some $1<k<n$. Using the definition of $h_{k}$ we get $\sum_{j=1}^{n} s_{j} g_{j}=0$, where $s_{k}=-z, s_{k+1}=0$. Applying the transposition $(k, k+1)$ for this and subtracting the result from the former equation we get $z\left(g_{k}-g_{k+1}\right)=0$. By symmetry $z h_{2}=z\left(g_{1}-g_{2}\right)=0$ follows. So for all $1<k<n$ the order of $h_{k}$ is $q$.

Now let $c=p / q$ and let $a$ be the order of $h_{n}$. Here $0=\sum_{j=1}^{n} q\left(g_{1}-g_{j}\right)=$ $q n g_{1}-q \sum_{j=1}^{n} g_{j}$, so $q h_{n}=q\left(F g_{1}-\sum_{j=1}^{n} g_{j}\right)=(q F-q n) h_{1}$. Thus using the 
independence of $h_{1}$ and $h_{n}$ we have $q h_{n}=0$ and $q(F-n) h_{1}=0$, proving $a \mid q$ and $p \mid q(F-n)$, that is, $c \mid F-n$.

Now $0=a h_{n}=a F g_{1}-a \sum_{j=1}^{n} g_{j}$. Applying the transposition $(1,2)$ to this equation and subtracting the result we get $0=a F\left(g_{1}-g_{2}\right)=a F h_{2}$, so $q \mid a F$. Let $b=q / a$; then we have $b \mid F$. Now let $d=\operatorname{gcd}(b, c)$. Then $d \mid F$ and $d \mid(F-n)$, and hence $d \mid n$ which is what we wanted to prove.

Conversely we show that if a finite Abelian group satisfies (i) and (ii), then it can be symmetrically generated.

Let $G=\left\langle h_{1}, h_{2}, \ldots, h_{n}\right\rangle$ be a canonical generating set of $G$, so that the $i$-th component of the product in (i) belongs to $h_{i}$.

We can solve the Diophantine equation $n=b x-c y$, because $\operatorname{gcd}(b, c) \mid n$. Let $F=b x$. Now $b \mid F$ and $c \mid F-n$. Let us define $g_{1}, \ldots, g_{n}$ in the following way:

$g_{1}=h_{1}$,

$g_{2}=h_{1}-h_{2}$

$g_{i}=h_{1}+h_{i-1}-h_{i} \quad(2<i<n)$,

$g_{n}=(F-n+1) h_{1}+h_{n-1}-h_{n}$.

We claim that $g_{1}, g_{2}, \ldots, g_{n}$ is a symmetric generating set of $G$.

The claim $G=\left\langle g_{1}, g_{2}, \ldots, g_{n}\right\rangle$ directly follows from the fact that the transformation matrix of $h_{i} \rightarrow g_{i}$,

$$
N=\left(\begin{array}{cccccc}
1 & 0 & 0 & 0 & \ldots & 0 \\
1 & -1 & 0 & 0 & \ldots & 0 \\
1 & 1 & -1 & 0 & \ldots & 0 \\
1 & 0 & 1 & -1 & \ldots & 0 \\
\vdots & \vdots & \vdots & \vdots & \ddots & \vdots \\
F-n+1 & 0 & 0 & 0 & \ldots & -1
\end{array}\right)
$$

has $\operatorname{det}(N)=(-1)^{n-1}$, so it can be inverted over $\mathbb{Z}$ (in fact, it is the inverse of the matrix $M)$.

To prove the symmetry, we only have to show that for all $1 \leq k<n$ the transposition $(k, k+1)$ brings all relations to a relation, because these transpositions generate the full symmetric group. Or, equivalently, we have to prove that if $\sum_{j=1}^{n} r_{j} g_{j}=0$, then we have $\sum_{j=1}^{k-1} r_{j} g_{j}+r_{k+1} g_{k}+r_{k} g_{k+1}+\sum_{j=k+2}^{n} r_{j} g_{j}=0$.

Using the definition of $g_{j}$ and the fact, that $h_{1}, h_{2}, \ldots, h_{n}$ is a canonical generating set of $G$ belonging to (i), we get the following reformulation of $\sum_{i=1}^{n} r_{i} y_{i}=0$ :

(1) $\sum_{i=1}^{n} r_{i}+(F-n) r_{n} \equiv 0(\bmod a b c)$,

(2) $r_{i+1}-r_{i} \equiv 0(\bmod a b)$ for all $1<i<n$ (so these coefficients are all congruent modulo $a b)$,

(3) $r_{n} \equiv 0(\bmod a)$.

All we have to prove is that this congruence system remains true if we apply the transposition $(k, k+1)$ to $\left(r_{1}, r_{2}, \ldots, r_{n}\right)$.

If $1<k<n-1$, then (1) and (2) trivially remains true, while (3) even formally does not change.

Let $k=n-1$. We know that $c \mid F-n$ and $a b \mid\left(r_{n}-r_{n-1}\right)$, so $(F-n)\left(r_{n}-r_{n-1}\right) \equiv 0$ $(\bmod a b c)$.

Subtracting this congruence from (1) we obtain $\sum_{i=1}^{n} r_{i}+(F-n) r_{n-1} \equiv 0$ $(\bmod a b c)$ which precisely means that (1) remains true. Now (2) remains true trivially, while (3) remains true because from $(2)$ we infer $r_{n-1} \equiv r_{n}(\bmod a)$. 
Lastly, if $k=1$, then we only have to check (2), the other two remaining true formally. We know that $b \mid F$ and $a \mid r_{n}$, so $a b \mid F r_{n}$. From this we get

$r_{n} \equiv-(F-1) r_{n} \equiv-(F-n) r_{n}-(n-1) r_{n} \equiv \sum_{i=1}^{n} r_{i}-(n-1) r_{n} \equiv r_{1} \quad(\bmod a b)$

by $(2)$, but then we have $r_{1} \equiv r_{n} \equiv r_{2}(\bmod a b)$ and so $(2)$ remains true.

\section{REFERENCES}

[Bee] M.J. Beetham, A set of generators and relations for the group $P S L(2, q), q$ odd, J. London Math. Soc. 3 (1971), 554-557. MR 44:2806

[BC] J.N. Bray, R.T. Curtis, A systematic approach to symmetric presentations II. Generators of order 3, Math. Proc. Cambridge Philos. Soc. 128 (2000), 1-20. MR 2000k:20032

[CHLR] C. Campbell, G. Havas, S. Linton, E. Robertson, Symmetric presentations and orthogonal groups: The atlas of finite groups: ten years on (Birmingham, 1995), 1-10, London Math. Soc. Lecture Note Ser., 249, Cambridge Univ. Press, Cambridge, 1998. MR 99m:20112

[CR] C.M. Campbell and E.F. Robertson, Some problems in group presentations, J. Korean Math. Soc. 19 (1983), 123-128. MR 84j:20030

[CRW] C.M. Campbell, E.F. Robertson and P.D. Williams, Efficient presentations of the groups $P S L(2, p) \times P S L(2, p), p$ prime, J. London Math. Soc. (2) 41 (1989), 69-77. MR 91g:20042

[Cox] H.S.M. Coxeter, Symmetrical definitions for the binary polyhedral groups, Proc. Sympos. Pure Math. 1 (1959), 64-87. MR 22:6850

[CM] H.S.M. Coxeter and W.O.J. Moser, Generators and relations for discrete groups, 4th edition (Springer, Berlin, 1979). MR 81a:20001

[Cu1] R.T. Curtis, Symmetric presentations. I. Introduction, with particular reference to the Mathieu groups $M_{12}$ and $M_{24}$, Groups, combinatorics \& geometry (London Math. Soc. Lecture Note Ser., 165, Cambridge Univ. Press, Cambridge, 1992), 380-396. MR 94b:20038

[Cu2] R.T. Curtis, Symmetric presentations. II. The Janko group $J_{1}$, J. London Math. Soc. (2) 47 (1993), 294-308. MR 94b:20039

[CHB] R.T. Curtis, A.M.A. Hammas, J.N. Bray, A systematic approach to symmetric presentations I. Involutory generators, Math. Proc. Cambridge Philos. Soc. 119 (1996), 23-34. MR 96k:20058

[Eme] W. Emerson, Groups defined by permutations of a single word, Proc. Amer. Math. Soc. 21 (1969), 386-390. MR 39:1530

[RC] E.F. Robertson and C.M. Campbell, Symmetric presentations, Group Theory (Walter de Gruyter, Berlin, New York, 1989), 497-506. MR 90a:20064

Alfréd Rényi Institute of Mathematics, Reáltanoda utca 13-15, H-1053, Budapest, HUNGARY

E-mail address: abert@renyi.hu 